Check for updates

Cite this: Chem. Commun., 2017, 53, 12426

Received 29th September 2017, Accepted 19th October 2017

DOI: $10.1039 / c 7 c c 07602 a$

rsc.li/chemcomm

\section{Optically active histidin-2-ylidene stabilised gold nanoparticles $\dagger$}

\author{
Adam J. Young, (D) a Christopher J. Serpell, (D) ${ }^{b}$ Jia Min Chin (D) $\star^{a}$ and \\ Michael R. Reithofer (D)*ac
}

\begin{abstract}
Drawing from the natural amino acid chiral pool, $L$ and $D$ histidines were utilized as chiral NHC ligands in the synthesis of NHC-stabilized chiroptical gold nanoparticles. Centrifugal size selection afforded monodisperse gold nanoparticles which display mirrored signals in CD spectroscopy.
\end{abstract}

Gold nanoparticles (AuNPs) have been exploited for centuries by artists for their vibrant colours, due to the strong interaction of light with electrons on the AuNP surface, giving rise to surface plasmon resonance (SPR). Recently, focus in AuNP research has been on their potential for various optoelectronic applications, alongside the development of reliable methods to tune the sizes, electronic structures and optical properties of these nanoparticles. ${ }^{1}$

The introduction of a two-phase synthesis exploiting thiols as AuNP stabilizers by Brust and co-workers ${ }^{2}$ served as an important breakthrough which facilitated the study and handling of AuNPs as well-defined soluble species. ${ }^{3-9}$ However, a drawback of the Brust method is that the S-Au bond may be strong but is not completely inert, limiting the long term stability of such AuNPs, which is essential for safe and reliable applications. ${ }^{10-12}$ As such, expanding the toolbox of functional surface anchors will provide unique opportunities for the construction of novel gold nanoparticle interfaces with greater stability.

$\mathrm{N}$-heterocyclic carbenes (NHCs) have emerged as attractive alternatives to thiol ligands, as the strong $\sigma$-donating property of NHC ligands affords stable metal-NHC complexes. Further, the synthetic accessibility ${ }^{13}$ of NHC ligands allows easy fine-tuning of their steric and electronic properties. Thus metal-NHC

\footnotetext{
${ }^{a}$ Gray Centre for Advanced Materials, School of Mathematics and Physical Sciences, University of Hull, Cottingham Road, Hull, East Riding of Yorkshire, HU6 7RX, UK.E-mail:j.chin@hull.ac.uk

${ }^{b}$ University of Kent, School of Physical Sciences, Ingram Building Canterbury, Kent, CT2 $7 \mathrm{NH}$, UK

${ }^{c}$ Institute of Inorganic Chemistry, University of Vienna, Faculty of Chemistry, Waehringer Strasse 42, 1090 Vienna, Austria.

E-mail: michael.reithofer@univie.ac.at

$\dagger$ Electronic supplementary information (ESI) available: Detailed synthetic procedures of compounds and characterization data. See DOI: 10.1039/c7cc07602a
}

compounds show promise for catalytic applications ${ }^{14}$ and also in the biological field. ${ }^{15,16}$ For similar reasons, NHCs have attracted much attention in the development of novel nanoparticles ${ }^{17-26}$ as it is estimated that the Au-NHC bond strength is about double that of a corresponding $\mathrm{Au}$-thiol bond, ${ }^{27}$ and recent reports show that NHC functionalized AuNPs and gold surfaces outperformed their thiol based counterparts with regards to thermal and chemical stability. ${ }^{24,27,28}$

Amongst AuNPs, those with chiral ligands are of particular interest due to their potential applications in optics, asymmetric catalysis and chiral discrimination. ${ }^{29}$ However, to date there have been only a few reports of nanoparticles stabilized by chiral NHC ligands. ${ }^{30,31}$ Despite the richness of the natural chiral pool, there are no reports of NHCs derived from chiral biomolecules as nanoparticle stabilizers. Therefore, we have focused here on utilizing an NHC derived from the inherently chiral amino acid histidine as the ligand for AuNPs (Scheme 1).

Histidine was selected as the chiral NHC precursor due to the ease of converting the imidazole side chain into an imidazolium derivative. ${ }^{15}$ Here we report a novel method of incorporating $\mathrm{N}$-BOC-histidine-methyl ester as a ligand to stabilize gold nanoparticle and the characterization of the resulting AuNPs stabilized with both enantiomers.

Methylation of commercially available $\mathrm{L}$ and $\mathrm{D}$ forms of BOCHis-OH, followed by a transmetallation procedure with $\mathrm{Ag}_{2} \mathrm{O}$ and $\mathrm{Au}\left(\mathrm{SMe}_{2}\right) \mathrm{Cl}$ yielded chlorido(1,3-dimethyl- $N$-BOC-O-methyl-L/ D-histidin-2-ylidene)gold(I), [2(L/D)], using a procedure reported by Albrecht et $a l .{ }^{15}$ The reduction of well-defined organometallic

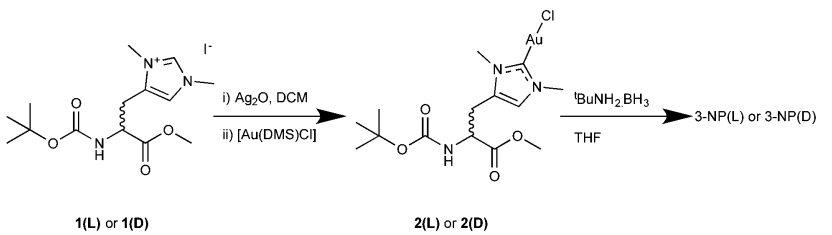

Scheme 1 Overview of synthetic routes employed to synthesise 3-NP(L) and 3-NP(D). 
gold(I) precursors containing ligands such as NHCs, phosphines or amines has proven to be a variable route to ligand-stabilised AuNPs. ${ }^{21-23,32-34}$ Therefore, to form AuNPs, ${ }^{t} \mathrm{BuNH}_{2} \cdot \mathrm{BH}_{3}$ was used as the reducing agent and added to solutions of either 2(L) or 2(D) in THF which resulted in the formation of a deep red solution, with no signs of aggregate formation. In order to isolate the pure AuNP, excess of reducing agent had to be removed. To do so, the reaction mixture was quenched with water, centrifuged and the nanoparticles were re-suspended in DCM resulting in a bright red solution of compound 3-NP(L) and 3-NP(D) respectively. The successful formation of AuNPs was indicated by the presence of a plasmon resonance band $(\mathrm{PRB})$ in the UV-vis spectrum at $517 \mathrm{~nm}$ for both 3-NP(L) and 3-NP(D) respectively (Fig. S23 and S25, ESI $\dagger$ respectively). Further, ${ }^{1} \mathrm{H}$ and ${ }^{13} \mathrm{C}$ NMR spectroscopy supported the binding of the histidine NHC ligand on the AuNP surface, whereby the ${ }^{13} \mathrm{C}$ NMR spectrum showed that the carbene NHC peak had shifted from $171.5 \mathrm{ppm}$ in the molecular species $2(\mathbf{L})$ to $184.0 \mathrm{ppm}$ in $3-\mathbf{N P}(\mathbf{L})$. The low field shift of the coordinating carbon clearly demonstrates a change in the chemical environment suggesting the successful formation of AuNPs. Similar changes in chemical shifts between the molecular and the nanoparticulate species have been recently reported Johnson et al. ${ }^{21}$ Further, the absence of a signal at about 137.0 ppm shows that no free ligand is detectable in solution.

TEM images (Fig. 1) of films obtained via drop-casting a solution of 3-NP(L) in THF and drying revealed the presence of non-agglomerated, spherical AuNPs with a bimodal size distribution throughout the sample, giving rise to a large size distribution whereby the AuNP diameter $\left(D_{\text {TEM }}\right)$ was $1.9 \pm 0.4 \mathrm{~nm}$ and $6.4 \pm 1.1 \mathrm{~nm}$ respectively [Fig. 1(a) and (b)]. Although a bimodal distribution was not observed for 3-NP(D), a relatively broad size distribution encompassing a similar size range was observed. This effect may be due to slight environmental differences during the reduction of 2(L/D). Pileni et al. have previously reported nanoparticle size selection through centrifugation to yield monodisperse NHC stabilised AuNPs. ${ }^{22}$ Therefore, 3-NP(L) was suspended in THF and centrifuged at $13500 \mathrm{rpm}$ to isolate the 'large' nanoparticles as a solid, with the expectation that the resultant supernatant should contain the 'small' particles. From the size selection technique exploited, it was possible to isolate monodisperse histidin-2-ylidene stabilised AuNPs of both enantiomers, as a black solid following centrifugation, yielding a red solution after re-suspension. TEM analysis revealed an average diameter $\left(D_{\text {TEM }}\right)$ of $5.0 \pm 0.6$ (12.0\%) nm [Fig. 1(c) and (d)]; whereas dynamic light scattering (DLS) analysis of a solution of 3-NP(L) in THF showed an average hydrodynamic diameter $\left(D_{\text {DLS }}\right)$ of $6.5 \pm 0.1 \mathrm{~nm}$. Similar 3-NP(D) showed an average hydrodynamic diameter ( $\left.D_{\text {DLS }}\right)$ of $5.5 \pm 0.1 \mathrm{~nm}$ and an average diameter $\left(D_{\text {TEM }}\right)$ of $5.3 \pm 0.8$ (15.8\%) nm [Fig. 1(g) and (h)]. However, isolation of the 'small' NPs via centrifugation was unsuccessful, as the supernatant still contained a mixture of different sized NPs (Fig. S27, ESI $\dagger$ ). UV-vis spectroscopy was conducted to determine the stability of 3-NP(L)/DCM solutions over 48 hours at room temperature. The PRB broadens over the two days, but the maxima peak remains at $517 \mathrm{~nm}$ (Fig. 2). Lowering of the baseline is observed, which
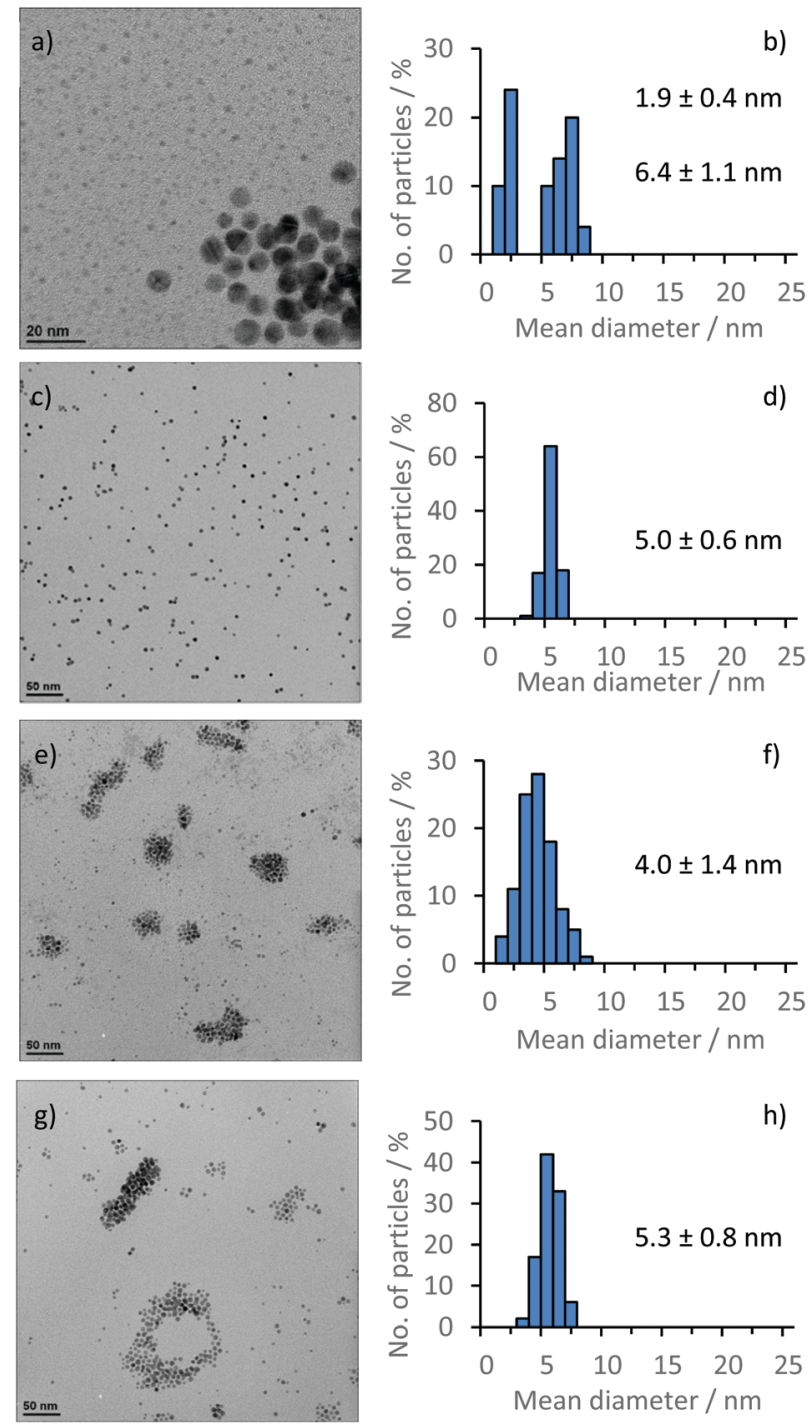

Fig. 1 TEM images of and size distribution graphs of; 3-NP(L), ( $a$ and $b$ ) pre-size selection and (c and d) post-size selection and 3-NP(D), (e and f) pre-size selection and ( $\mathrm{g}$ and $\mathrm{h}$ ) post-size selection.

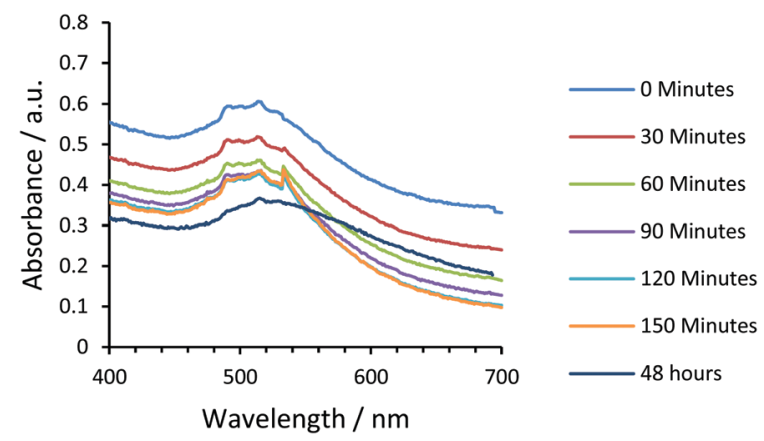

Fig. 2 UV-vis spectra of $1 \mathrm{mg} \mathrm{mL}^{-1}$ of 3-NP(L) in DCM measuring absorbance over a period of time.

could be due to solvent evaporation, and the broadening of the PRB may indicate that the AuNPs start to aggregate or degrade under these conditions. 


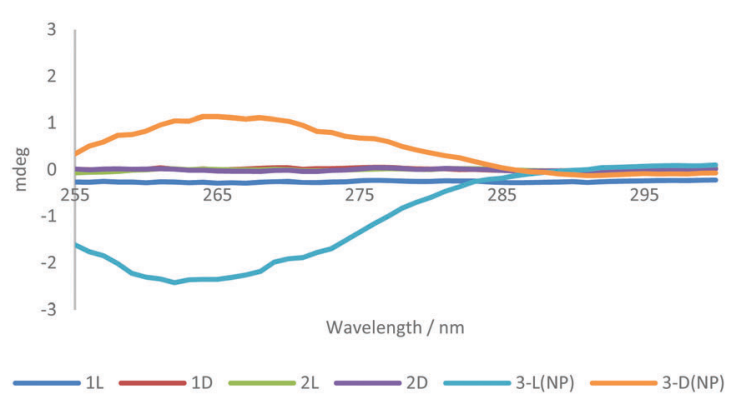

Fig. 3 CD spectra of synthesised compounds dissolved dispersed in DCM.

Due to the chiral nature of the histidin-2-ylidene derivative gold complex [2(L) or (D)], we surmised that the resulting AuNPs may display optical activity. Therefore, subsequent to centrifugal size selection, the optical activity of 3-NP(L) and 3-NP(D) was analysed using circular dichroism (CD) spectroscopy with DCM as the solvent (Fig. 3). The dichroic effects are seen in the ligand region of the spectrum, indicating that there is chiral ordering of the amino acids on the AuNP surface. The role of the nanoparticle is crucial, since in its absence neither the imidazolium salt nor molecular $\mathrm{Au}-\mathrm{NHC}$ complex shows significant optical activity - only when the NHC ligands are concentrated on the surface of the NP is this effect seen. Typically, small chiral molecules that lack a secondary structure do not display optical activity in the CD spectrum above $230 \mathrm{~nm}$ wavelengths. For example, the CD activity of single amino acids in water was only observed below $230 \mathrm{~nm}$, which is the threshold of the DCM solvent we utilized. However, packing of small chiral molecules into an ordered structure affords signals in the CD spectrum. ${ }^{35}$ In this case, the NPs appear to fix the histidine ligands in a certain conformation that gives rise to optical activity associated with supramolecular ordering rather than singular molecular chirality. We attribute the minor deviations from perfect mirroring of spectra between $\mathrm{L}^{-}$and D-variants to differential absorption flattening effects, which become more significant at higher chromophore concentrations. ${ }^{36}$ Thermogravimetric analysis showed that 3-NP(L) and 3-NP(D) possessed $26.4 \%$ and $38.7 \%$ organic component by weight respectively (Fig. S32 and S33, $\mathrm{ESI} \dagger$ respectively). The higher ligand coverage in 3-NP(D) results in greater absorption flattening, leading to decreased signal intensity and a slight red shift of the peak. Similar phenomena for chiral complexes $^{37}$ and peptides have also been observed by others. ${ }^{38}$ Further, the metallation procedure utilised may lead to partial ligand racemization, giving a slight shift of the CD spectra of 3-NP(L) and 3-NP(D). ${ }^{39}$ CD spectroscopy showed no signal in the PRB region, indicating that chirality is limited to the NHC ligands, and that the nanoparticle lattice is achiral.

In an attempt to make the NPs water soluble, deprotection of the BOC group was attempted. Whereby, 3-NP(L), N-BOCprotected nanoparticles were suspended in THF and four drops of concentrated $\mathrm{HCl}$ acid were added. The dispersion goes from a ruby red colour to colourless with concomitant formation of black aggregates. The aggregates were isolated via centrifugation and unsuccessful attempts to disperse the aggregates in a variety of aprotic and protic solvents (i.e. methanol, ethanol, THF, DCM, chloroform, toluene, water, dioxane) showed that the nanoparticles had undergone irreversible aggregation. A small portion of this sample was drop-cast onto a TEM grid to reveal a polydisperse sample with a variety of different morphologies (Fig. S29, ESI $\dagger$ ). As water soluble nanoparticles are desirable for biological applications, future work will include adaptation of this procedure to synthesise monodisperse particles that are water soluble, with functional groups to facilitate self-assembly and post synthetic modification.

In summary, we have drawn from the natural chiral pool to create chiral, histidine-derived Au-NHC complexes chlorido(1,3-dimethyl- $N$-BOC-O-methyl-L/D-histidin-2-ylidene)gold(I), [2(L/D)]. The chiral complexes were reduced by ${ }^{t} \mathrm{BuNH}_{2} \cdot \mathrm{BH}_{3}$ to afford AuNPs showing a bimodal size distribution. Nanoparticle size selection was carried out by centrifugation of the NP suspensions, allowing isolation of monodisperse nanoparticles. CD spectroscopy showed optical activity of the AuNPs arising from the chiral NHC ligands but not for the Au-NHC complexes, indicating that packing of the ligands on the nanoparticle surface is important for the chiral effects observed. The nanoparticles are dispersible in aprotic organic solvents such as THF and DCM and the solutions are stable over 48 hours at room temperature. This work demonstrates for the first time a way to utilize amino-acid derived NHC ligands for nanoparticle stabilization, and will find potential uses in the creation of other nanoparticle systems displaying optical activity. Such systems could be exploited for use in asymmetric catalysis, chiral sensing, optoelectronics or drug delivery and further work will be employed to make water soluble variants.

M. R. R. thanks the University of Vienna for a start up grant. M. R. R. and J. C. thank the University of Hull for providing the financial support to carry out this work. A. J. Y. thanks the University of Hull for the provision of a University Scholarship C. J. S. thanks the University of Kent for financial support.

\section{Conflicts of interest}

The authors declare no conflict of interest.

\section{Notes and references}

1 U. Kreibig and M. Vollmer, Optical properties of metal clusters, Springer, Berlin, Heidelberg, 1995, vol. 25.

2 M. Brust, M. Walker, D. Bethell, D. J. Schiffrin and R. Whyman, Chem. Commun., 1994, 801-802.

3 W. P. Wuelfing, S. M. Gross, D. T. Miles and R. W. Murray, J. Am. Chem. Soc., 1998, 120, 12696-12697.

4 E. Locatelli, I. Monaco and M. Comes Franchini, RSC Adv., 2015, 5, 21681-21699.

5 S. T. Kim, K. Saha, C. Kim and V. M. Rotello, Acc. Chem. Res., 2013, 46, 681-691.

6 J. Gao, X. Huang, H. Liu, F. Zan and J. Ren, Langmuir, 2012, 28, 4464-4471.

7 E. Hao, R. C. Bailey, G. C. Schatz, J. T. Hupp and S. Li, Nano Lett., 2004, 4, 327-330.

8 S. J. Hsu, K. M. Hsu, M. K. Leong and I. J. B. Lin, Dalton Trans., 2008, 1924-1931.

9 S. Kundu, A. Pal, S. K. Ghosh, S. Nath, S. Panigrahi, S. Praharaj and T. Pal, Inorg. Chem., 2004, 43, 5489-5491.

10 M. M. Maye, W. Zheng, F. L. Leibowitz, N. K. Ly and C. J. Zhong, Langmuir, 2000, 16, 490-497. 
11 N. Bhatt, P.-J. J. Huang, N. Dave and J. Liu, Langmuir, 2011, 27, 6132-6137.

12 L. Srisombat, A. C. Jamison and T. R. Lee, Colloids Surf., A, 2011, 390, 1-19.

13 S. Díez-González and S. P. Nolan, Coord. Chem. Rev., 2007, 251, 874-883.

14 D. M. Flanigan, F. Romanov-Michailidis, N. A. White and T. Rovis, Chem. Rev., 2015, 115, 9307-9387.

15 F. Schmitt, K. Donnelly, J. K. Muenzner, T. Rehm, V. Novohradsky, V. Brabec, J. Kasparkova, M. Albrecht, R. Schobert and T. Mueller, J. Inorg. Biochem., 2016, 163, 221-228.

16 A. M. Al-Majid, S. Yousuf, M. I. Choudhary, F. Nahra and S. P. Nolan, ChemistrySelect, 2016, 1, 76-80.

17 K. Salorinne, R. W. Y. Man, C.-H. Li, M. Taki, M. Nambo and C. M. Crudden, Angew. Chem., Int. Ed., 2017, 56, 6198-6202.

18 A. Ferry, K. Schaepe, P. Tegeder, C. Richter, K. M. Chepiga, B. J. Ravoo and F. Glorius, ACS Catal., 2015, 5, 5414-5420.

19 P. Lara, L. M. Martínez-Prieto, M. Roselló-Merino, C. Richter, F. Glorius, S. Conejero, K. Philippot and B. Chaudret, Nano-Struct. Nano-Objects, 2016, 6, 39-45.

20 C. J. Serpell, J. Cookson, A. L. Thompson, C. M. Brown and P. D. Beer, Dalton Trans., 2013, 42, 1385-1393.

21 M. J. MacLeod and J. A. Johnson, J. Am. Chem. Soc., 2015, 137, 7974-7977.

22 S. Roland, X. Ling and M.-P. Pileni, Langmuir, 2016, 32, 7683-7696.

23 J. Vignolle and T. D. Tilley, Chem. Commun., 2009, 7230-7232.

24 C. M. Crudden, J. H. Horton, I. I. Ebralidze, O. V. Zenkina, A. B. McLean, B. Drevniok, Z. She, H.-B. Kraatz, N. J. Mosey, T. Seki, E. C. Keske, J. D. Leake, A. Rousina-Webb and G. Wu, Nat. Chem., 2014, 6, 409-414.
25 G. Wang, A. Rühling, S. Amirjalayer, M. Knor, J. B. Ernst, C. Richter, H.-J. Gao, A. Timmer, H.-Y. Gao, N. L. Doltsinis, F. Glorius and H. Fuchs, Nat. Chem., 2016, 9, 152-156.

26 J. Crespo, Y. Guari, A. Ibarra, J. Larionova, T. Lasanta, D. Laurencin, J. M. López-de-Luzuriaga, M. Monge, M. E. Olmos and S. Richeter, Dalton Trans., 2014, 43, 15713-15718.

27 P. Pyykk and N. Runeberg, Chem. - Asian J., 2006, 1, 623-628.

28 X. Ling, N. Schaeffer, S. Roland and M.-P. Pileni, Langmuir, 2015, 31, 12873-12882.

29 C. Gautier and T. Bürgi, ChemPhysChem, 2009, 10, 483-492.

30 K. V. S. Ranganath, J. Kloesges, A. H. Schäfer and F. Glorius, Angew. Chem., Int. Ed., 2010, 49, 7786-7789.

31 L. M. Martínez-Prieto, A. Ferry, P. Lara, C. Richter, K. Philippot, F. Glorius and B. Chaudret, Chem. - Eur. J., 2015, 21, 17495-17502.

32 X. Lu, M. S. Yavuz, H.-Y. Tuan, B. A. Korgel and Y. Xia, J. Am. Chem. Soc., 2008, 130, 8900-8901.

33 G. Schmid, B. D. Alexander, J. Barthelmes, A. M. Mueting and L. H. Pignolet, Inorg. Synth., 1990, 214-218.

34 W. W. Weare, S. M. Reed, M. G. Warner and J. E. Hutchison, J. Am. Chem. Soc., 2000, 122, 12890-12891.

35 N. Amdursky and M. M. Stevens, ChemPhysChem, 2015, 16, 2768-2774.

36 B. A. Wallace and C. L. Teeters, Biochemistry, 1987, 26, 65-70.

37 E. Castiglioni, S. Abbate, G. Longhi, R. Gangemi, R. Lauceri and R. Purrello, Chirality, 2007, 19, 642-646.

38 G. Manzo, M. A. Scorciapino, P. Wadhwani, J. Bürck, N. Pietro Montaldo, M. Pintus, R. Sanna, M. Casu, A. Giuliani, G. Pirri, V. Luca, A. S. Ulrich and A. C. Rinaldi, PLoS One, 2015, 10, e0116379.

39 A. Monney, E. Alberico, Y. Ortin, H. Müller-Bunz, S. Gladiali and M. Albrecht, Dalton Trans., 2012, 41, 8813. 